\title{
The Next Generation Sky Survey and the Quest for Cooler Brown Dwarfs
}

\author{
J. Davy Kirkpatrick \\ Infrared Processing and Analysis Center, California Institute of \\ Technology, Pasadena, CA 91125, USA
}

\begin{abstract}
The Next Generation Sky Survey (NGSS) is a proposed NASA MIDEX mission to map the entire sky in four infrared bandpasses - 3.5, 4.7, 12, and $23 \mu \mathrm{m}$. The seven-month mission will use a $50-\mathrm{cm}$ telescope and four-channel imager to survey the sky from a circular orbit above the Earth. Expected sensitivities will be half a million times that of COBE/DIRBE at 3.5 and $4.7 \mu \mathrm{m}$ and a thousand times that of IRAS at 12 and $23 \mu \mathrm{m}$. NGSS will be particularly sensitive to brown dwarfs cooler than those presently known. Deep absorption in the methane fundamental band at $3.3 \mu \mathrm{m}$ and a predicted 5- $\mu \mathrm{m}$ overluminosity will produce uniquely red 3.5-to- $4.7 \mu \mathrm{m}$ colors for such objects. For a limiting volume of $25 \mathrm{pc}$, NGSS will completely inventory the Solar Neighborhood for brown dwarfs as cool as Gl 229B. At $10 \mathrm{pc}$, the census will be complete to $500 \mathrm{~K}$. Assuming a field mass function with $\alpha=1$, there could be one or more brown dwarfs warmer than $150 \mathrm{~K}$ lying closer to the Sun than Proxima Centauri and detectable primarily at NGSS wavelengths. NGSS will enable estimates of the brown dwarf mass and luminosity functions to very cool temperatures and will provide both astrometric references and science targets for NGST.
\end{abstract}

\section{Introduction}

Large-area surveys such as the Two Micron All Sky Survey (2MASS; see other contribution by Kirkpatrick) and the Sloan Digital Sky Survey (SDSS; see contribution by Covey) have revolutionized the field of brown dwarf science. However, these surveys are able to probe only to temperatures near $1000 \mathrm{~K}$ at a distance of $10 \mathrm{pc}$, which leaves the realm of cooler brown dwarfs almost entirely unexplored. Fortunately, the Next Generation Sky Survey (NGSS) has been proposed in a large part to characterize cooler brown dwarfs in the Solar Neighborhood. In $\S 2$ the NGSS project will be briefly described. Its role in the future of brown dwarf studies is highlighted in $\S 3$, and the current status of the mission is given in $\S 4$.

\section{An Overview of the NGSS Mission}

NGSS is a proposed seven-month mission to survey the entire sky between 3.5 and $23 \mu \mathrm{m}$. The $50-\mathrm{cm}$ telescope flies is a circular orbit $500 \mathrm{~km}$ above the Earth. It is equipped with a four-channel imager comprised of $\mathrm{HgCdTe}$ and 


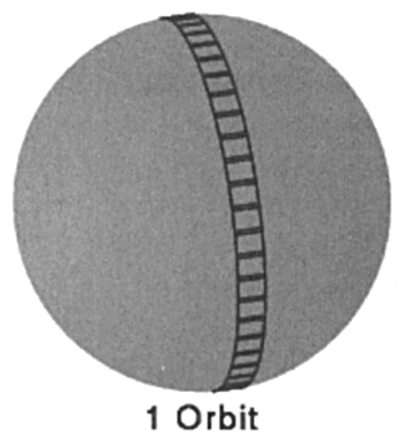

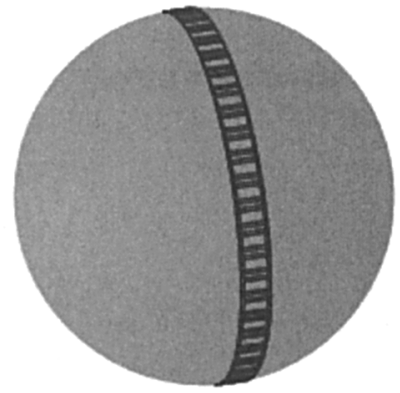

2 Consecutive Orbits

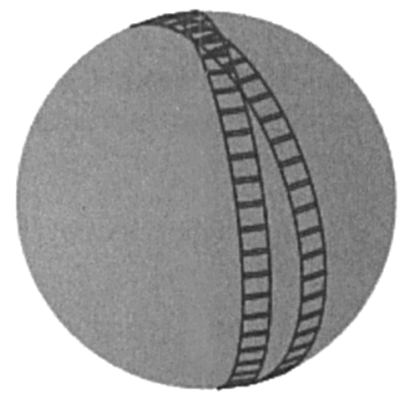

2 Orbits 20 Days Apart

Figure 1. Examples of the orbital scanning pattern. For clarity, the size of the frames has been exaggerated and the number of frames per orbit reduced.

$\mathrm{Si}$ As $1024 \times 1024$ arrays to survey the sky at the four wavelengths given in Table 1. The resolution of the telescope plus re-imaging optics is $\sim 5^{\prime \prime}$ except at 12 and $23 \mu \mathrm{m}$ where it is near the diffraction limit of $6^{\prime \prime}$ and $12^{\prime \prime}$, respectively. The actual field of view of the detectors is $38^{\prime} \times 38^{\prime}$.

\begin{tabular}{cccc} 
Table 1. & \multicolumn{3}{c}{ Wavelengths and Sensitivities Probed by NGSS } \\
\cline { 2 - 4 } $\begin{array}{c}\text { Wavelength } \\
(\mu \mathrm{m})\end{array}$ & Note & $\begin{array}{c}\text { Sensitivity } \\
(\mu \mathrm{Jy})\end{array}$ & $\begin{array}{c}\text { Sensitivity } \\
(\mathrm{mag})\end{array}$ \\
\hline 3.5 & $\sim \mathrm{L}$-band & $\sim 24$ & $\sim 17.6$ \\
4.7 & $\sim \mathrm{M}$-band & $\sim 30$ & $\sim 16.8$ \\
12 & $\sim \mathrm{N}$-band & $\sim 131$ & $\sim 13.3$ \\
23 & $\ldots$ & $\sim 414$ & $\sim 10.8$ \\
\hline
\end{tabular}

NGSS will cover the sky from a sun-synchronous polar orbit with a 6 am/6 pm ascending node like IRAS. As it circles the earth, the telescope performs one revolution per orbit so that it is always pointing away from the earth. A scanning mirror freezes a $38^{\prime} \times 38^{\prime}$ field-of-view on the detectors every 8.8 seconds for a total integration time per frame of $6.6 \mathrm{sec}$. The rate of reset for the scanning mirror is chosen so that there is a small overlap between consecutive frames, and a great circle perpendicular to the Earth-Sun line is scanned every orbit. There is a large overlap from one orbit to the next with the maximal overlap occurring at the ecliptic poles. A cartoon of the scanning procedure is shown in Figure 1.

In August, 1998, NGSS was first proposed to NASA as a mission in the Medium-class Explorer (MIDEX) program. Although selected as one of five missions for Phase-A study in January, 1999, it was not selected as one of the winners in the final round in October, 1999. NGSS was re-proposed as a MIDEX mission in October, 2001, and was one of four missions selected for Phase-A study in April, 2002. 


\section{The NGSS Contribution to Brown Dwarf Science}

Approximate sensitivities (away from the ecliptic plane) are listed in Table 1. In the two short wavelength bands, NGSS is 500,000 times deeper than COBE/DIRBE. At its two long wavelength bands, it is 1,000 times deeper than the short wavelength bands of IRAS. For the purposes of brown dwarf science, it is the 3.5- and 4.7- $\mu \mathrm{m}$ bands that are the most useful. Here NGSS is exploring wavelengths intermediate between those of 2MASS and IRAS, it probes them deeply, and it covers the entire sky.

Figure 2 shows a schematic diagram of currently recognized brown dwarf types. Thanks to large-area surveys such as 2MASS and SDSS, a large number of field late-M, L, and T dwarfs are now recognized. According to the models of Burrows et al. (1997), for an age of 1 Gyr an M8 dwarf would lie near the stellar/substellar border of $75 \mathrm{M} J u p$, a mid-L dwarf would have a mass of $\sim 65$ $\mathrm{M}_{J u p}$, and a mid- $\mathrm{T}$ would have a mass of $\sim 30 \mathrm{M}_{J u p}$. The models predict effective temperatures of $2200 \mathrm{~K}, 1700 \mathrm{~K}$, and $1200 \mathrm{~K}$ for these same objects. The 2MASS and SDSS surveys are sensitive to mid-T dwarfs only out to about $10 \mathrm{pc}$, and the coolest object so far discovered by either survey has an effective temperature of only $\sim 750 \mathrm{~K}$ (Gliese $570 \mathrm{D}$ at $d=5.9 \mathrm{pc}$; Burgasser et al. 2000).

The peak emission of even colder brown dwarfs lies squarely in the wavelength regime in which NGSS operates. If such objects radiated like blackbodies, a $500-\mathrm{K}$ brown dwarf would have its peak emission at $5.8 \mu \mathrm{m}$ and a $200-\mathrm{K}$ object would peak near $14.5 \mu \mathrm{m}$. However, the complex chemistry of these atmospheres results in a spectral energy distribution far different from a standard blackbody. Using model atmosphere calculations from Burrows et al. (1997), it is found that the peak flux for brown dwarfs with $1000 \mathrm{~K} \lesssim \mathrm{T}_{\text {eff }} \lesssim 200 \mathrm{~K}$ is around $4.7 \mu \mathrm{m}$. $^{1}$ The reason is that this is one of the few near-infrared wavelengths at which a major absorption band is not found $-\mathrm{H}_{2} \mathrm{O}, \mathrm{CH}_{4}$, and $\mathrm{H}_{2}$ provide major sources of opacity between 1 and $4 \mu \mathrm{m}$, and $\mathrm{H}_{2} \mathrm{O}, \mathrm{CH}_{4}$, and $\mathrm{NH}_{3}$ provide more opacity between 5 and $9 \mu \mathrm{m}$.

The predicted spectral energy distribution for a cool brown dwarf is shown in Figure 3. Specifically, this is a Burrows et al. (1997) model for a brown dwarf with $\mathrm{T}_{\text {eff }}=500 \mathrm{~K}$ and age $\approx 1$ Gyr. Locations and breadths of the NGSS photometric bands are shown by the shading in the figure. Note the flux peak between 4.0 and $5.0 \mu \mathrm{m}$ and the dominant absorption bands to either side of this. Cool brown dwarf candidates can thus be selected from the lists of NGSS detections by choosing those sources with extremely red $[3.5 \mu \mathrm{m}]-[4.7 \mu \mathrm{m}]$ colors.

Brown dwarfs with $\mathrm{T}_{\text {eff }}>800 \mathrm{~K}$, seen at increasingly larger distances from the Sun, will become non-detections at 12 and $23 \mu \mathrm{m}$ before they are undetectable in the other two NGSS bands. Brown dwarfs with $\mathrm{T}_{\text {eff }}<800 \mathrm{~K}$ drop out first in the 3.5 and $23 \mu \mathrm{m}$ bands. Requiring a candidate to be detected in two bands (i.e., so there would be a solid NGSS-determined color) gives, as a function of brown dwarf $\mathrm{T}_{e f f}$, the distance limits shown in the second column of

\footnotetext{
${ }^{1}$ As one might expect, the exact wavelength of this peak is a function of $\mathrm{T}_{\text {eff }}$ and gravity since it is shaped by the wings of strong molecular absorption bands sculpting it on both sides. Although the wavelength of the peak can shift either blueward or redward of the quoted value by a few tenths of a micron, $4.7 \mu \mathrm{m}$ is a reasonable median value for the peak over a large range of physical parameters (Burrows, priv. comm.).
} 


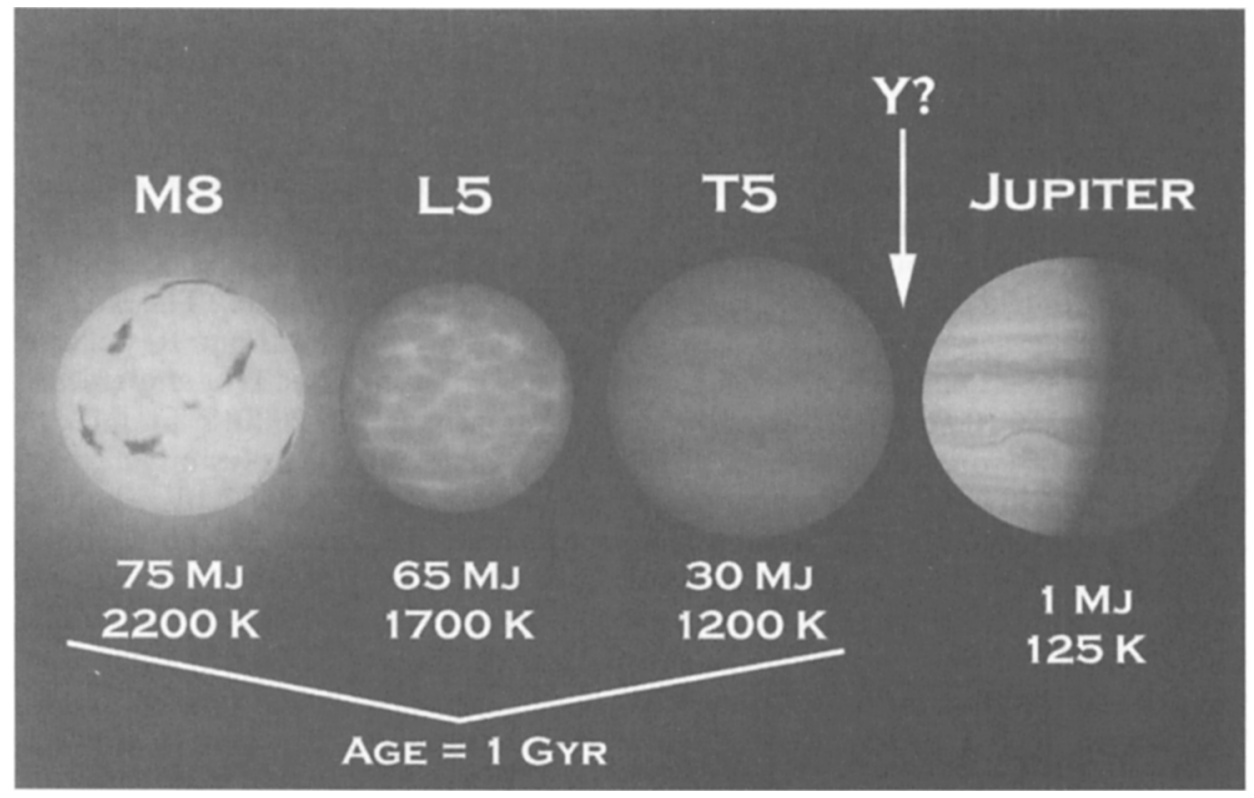

Figure 2. An artist's rendition comparing three flavors of brown dwarfs with Jupiter. All are plotted on the same size scale and the three brown dwarfs are chosen to have ages of $1 \mathrm{Gyr}$. On the far left is shown an M8 dwarf at the stellar/substellar border followed by a mid$\mathrm{L}$ dwarf and a mid-T dwarf. Temperatures and masses, derived from theory, are listed on the figure. Note the wide gap in mass and temperature between the mid-T dwarf and the planet Jupiter. It is within this gap that NGSS will have its greatest impact for brown dwarf research, uncovering even cooler objects (which we might call "Y dwarfs" if a new feature appears that is not characteristic of $\mathrm{T}$ dwarfs; e.g., the reshaping of the spectrum when $\mathrm{H}_{2} \mathrm{O}$ clouds form below $T_{\text {eff }} \approx 400 \mathrm{~K}$ ) and revealing just how prevalent brown dwarfs are in the solar vicinity. 


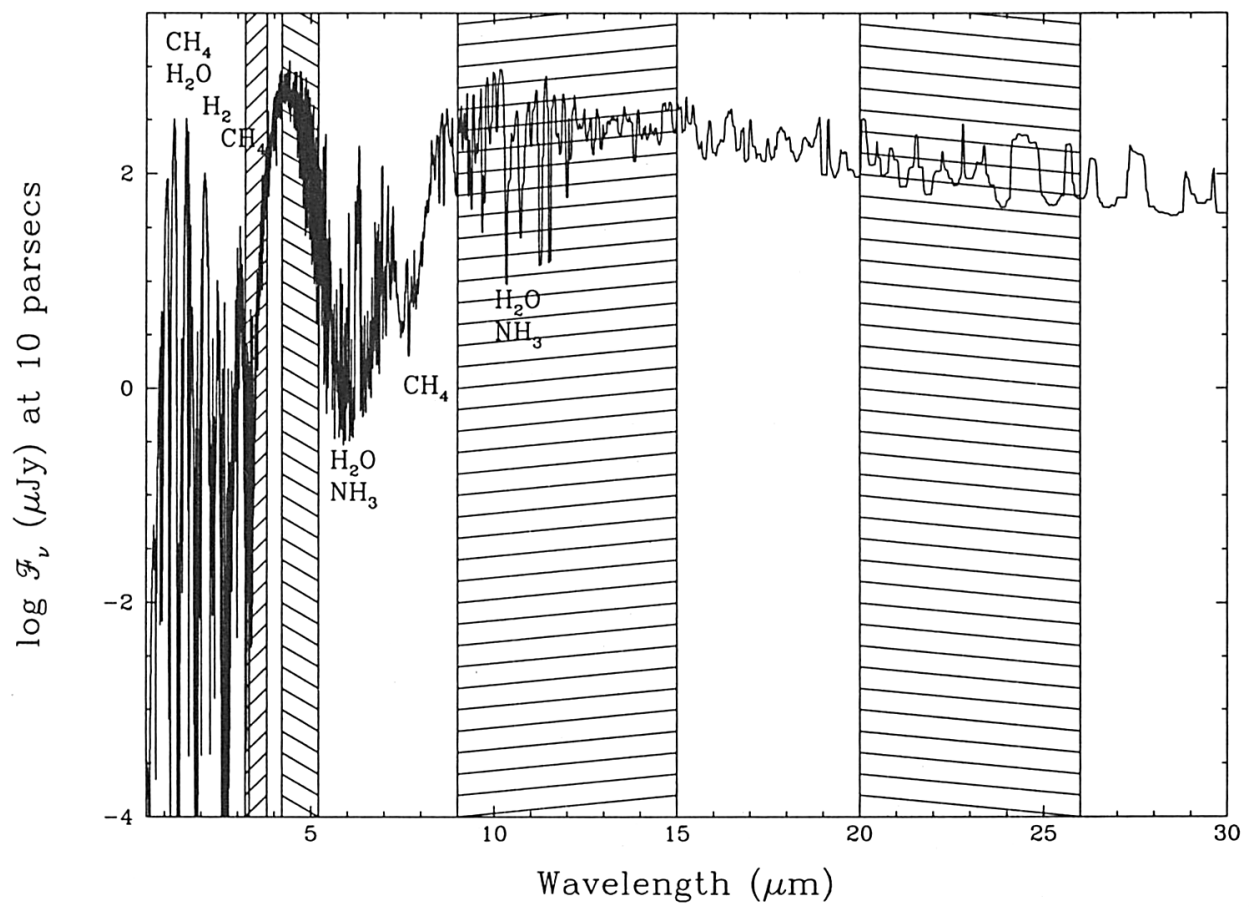

Figure 3. A Burrows et al. model of a $500 \mathrm{~K}$ brown dwarf with an age of $\sim 1$ Gyr. Major absorption bands by methane, water, ammonia, and molecular hydrogen are shown. The four filter bandpasses of NGSS are shown by the shaded regions. 
Table 2. If we resort to color limits and require that a source be detected in only a single NGSS band (which is always the $4.7 \mu \mathrm{m}$ filter for cool brown dwarfs), we could in principle probe to the distance limits given in the third column of Table 2 .

\begin{tabular}{ccc} 
Table 2. & \multicolumn{3}{c}{ Brown Dwarf Detection Limits for NGSS } \\
\cline { 2 - 3 } $\begin{array}{c}\text { Temperature of } \\
\text { Brown Dwarf } \\
\text { (K) }\end{array}$ & $\begin{array}{c}\text { Distance Limit } \\
\text { Probed, 2-band } \\
\text { Detections (pc) }\end{array}$ & $\begin{array}{c}\text { Distance Limit } \\
\text { Probed, 1-band } \\
\text { Detections (pc) }\end{array}$ \\
\hline 1200 & 32 & 72 \\
900 & 22 & 58 \\
750 & 16 & 41 \\
450 & 9 & 23 \\
300 & 6 & 10 \\
150 & 2 & 5 \\
\hline
\end{tabular}

To determine the number of cool brown dwarfs that NGSS might uncover, we are forced to extrapolate based on the rather poorly determined (at present) field brown dwarf mass function. Reid et al. (1999) made the first such determination using early discoveries of $\mathrm{L}$ and $\mathrm{T}$ dwarfs from 2MASS and DENIS. They assumed that the mass function is described by a power law $\left(\Psi(M)=d N / d M \propto M^{\alpha}\right)$ and that the birthrate of brown dwarfs has been constant over the lifetime of the Galaxy. Assuming various values of $\alpha$, they used the evolutionary models of Burrows et al. (1997) to assess the makeup of a complete collection of brown dwarfs in the Solar Neighborhood if that value of $\alpha$ were the correct one. Then using the search criteria employed in finding the earliest L dwarf discoveries from 2MASS (Kirkpatrick et al. 1999), they ran Monte Carlo simulations on the collections to derive typical "observed samples" of brown dwarfs. The set of models most closely representing the numbers and composition of the actual L dwarfs found in the 2MASS sample had $1<\alpha<2$, with the lower values of the exponent being preferred. It should be noted that Chabrier (2002) also finds a value close to $\alpha=1$ to be the best fit, which furthermore is in agreement with the value for $\alpha$ inferred from brown dwarf discoveries in young open clusters.

This work is based on observations of higher-mass and/or younger brown dwarfs since those are the only ones to which current surveys are sensitive. If we extrapolate to lower masses - into the mass regime where NGSS can probe - we find that there should be at least $\sim 200$ brown dwarfs with $M>10 M_{J u p}$ within $8 \mathrm{pc}$ of the Sun. A volume limited sample should contain 1.6 times as many brown dwarfs with $\mathrm{T}_{\text {eff }}=500 \mathrm{~K}$ than $1000 \mathrm{~K}$, and ones at $250 \mathrm{~K}$ should outnumber those at $500 \mathrm{~K}$ by a factor of 2.0 . The consequence of these results on the makeup of the Solar Neighborhood is shown graphically in Figure 4.

This implies that the nearby "stellar" census is at least $50 \%$ incomplete at present since most of the brown dwarfs are missing. Statistically speaking, then, there is a good chance that the nearest brown dwarf lies closer to the Sun than Proxima Centauri at $d=1.3$ pc. If we again assume $\alpha=1$ and take as 


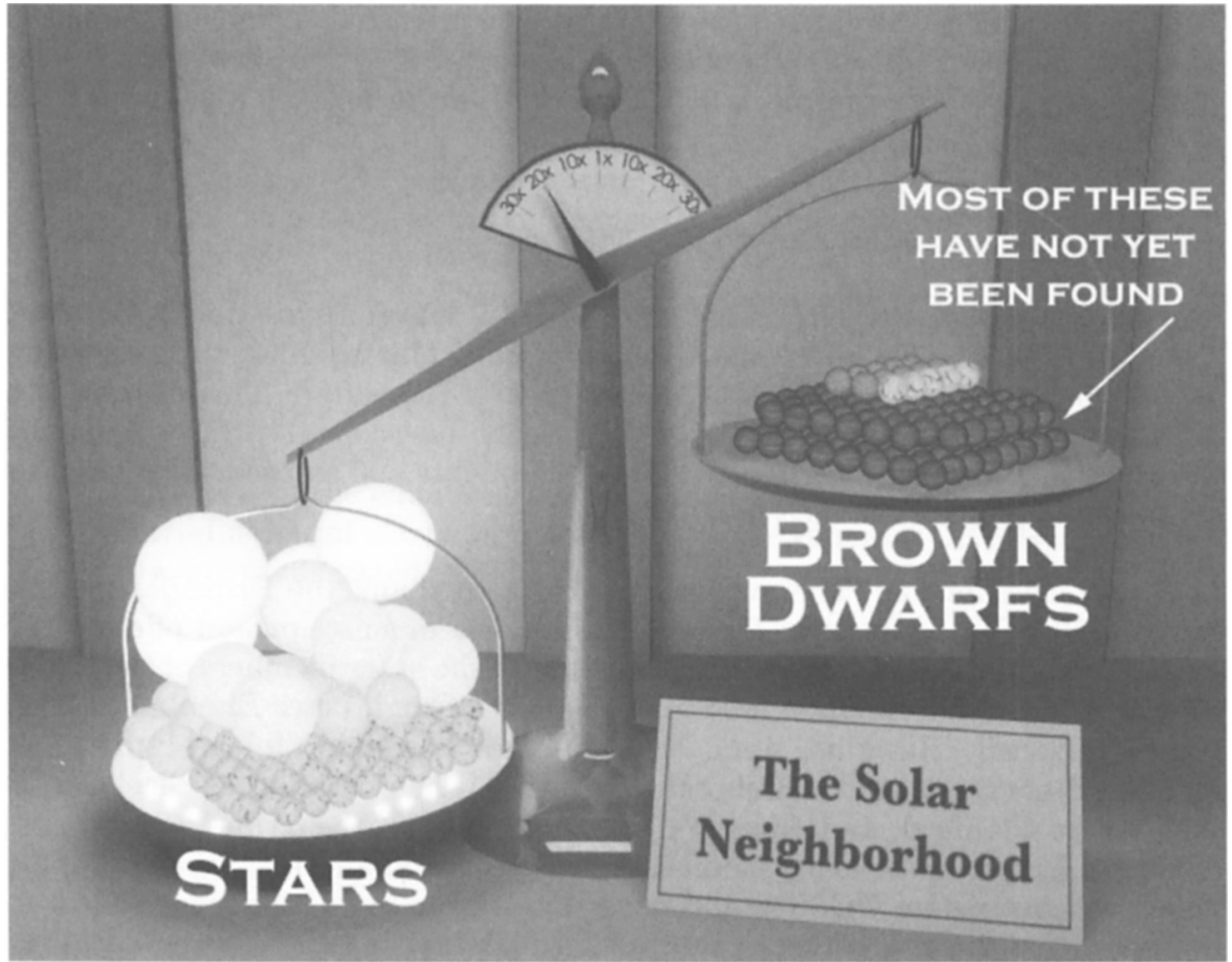

Figure 4. The makeup of a typical slice of the Milky Way - specifically the sample of nearby stars with $d<8$ pc and $\delta>-30^{\circ}$. Stars are shown on the left and brown dwarfs (with mass $>10 \mathrm{M}_{J u p}$ ) on the right. Going from the lightest colored, largest stars to the darkest, smallest ones, there are $4 \mathrm{~A}$ stars, $1 \mathrm{~F}$ star, $5 \mathrm{G}$ stars (one of which is the Sun itself), $22 \mathrm{~K}$ stars, and $87 \mathrm{M}$ stars. Amongst the collection of stars on the left are also 9 white dwarfs, shown as small, bright white dots. On the right scale are brown dwarfs, composed of a few $\mathrm{M}$ and $\mathrm{L}$ dwarfs along with lots of much darker T dwarfs or cooler objects, most of which are so cool that even 2MASS and SDSS cannot detect them. Despite the fact that there are at least as many brown dwarfs as stars, brown dwarfs appear to be responsible for only a small fraction of the total mass, as the scale shows. 
a worst-case scenario $1 M_{J u p}$ to be the cutoff mass for brown dwarf formation, then the median $\mathrm{T}_{\text {eff }}$ of the present-day brown dwarf field population should be $\sim 150 \mathrm{~K}$. As Table 2 shows, such an object is detectable by NGSS in two bands out to $d=2 \mathrm{pc}$. Superlatives and possible future press releases aside, within $10 \mathrm{pc}$ of the Sun NGSS can perform a complete census of objects down to at least $35 M_{J u p}$ since the oldest brown dwarfs (at age $\approx 10 \mathrm{Gyr}$ ) of this mass have cooled to $500 \mathrm{~K}$. This mass function will be sampled to even lower masses for younger objects. For example, a $500 \mathrm{~K}$ brown dwarf at age $100 \mathrm{Myr}$ has a mass of $5 M_{\text {Jup }}$.

\section{Current Status of the NGSS Mission}

Phase-A studies for the current round of MIDEX selections are due in October, 2002, and NASA will select the winners around March, 2003. If successful, NGSS would launch sometime in 2007 or shortly thereafter. This mission is a necessary precursor to the Next Generation Space Telescope (NGST) - hence the similarity in names - as it will provide science targets and astrometric references for the NGST, due to launch in 2010.

Acknowledgments: The author would like to thank the Principal Investigator of NGSS, Ned Wright, for proofreading the manuscript and offering the graphic used in Figure 1. The author also thanks the other members of the NGSS Science Team (Andrew Blain, Martin Cohen, Roc Cutri, Peter Eisenhardt, Nick Gautier, Isabelle Hawkins, Tom Jarrett, Carol Lonsdale, John Mather, Ian McLean, Robert McMillan, Deborah Padgett, Michael Ressler, Michael Skrutskie, Adam Stanford, and Russ Walker) for providing their time and support to the project, and the many members of the technical and managerial support teams without whom NGSS would never fly. The artistic renditions shown in Figures 2 and 4 , both of which are publicly available at the Archive of $\mathrm{M}, \mathrm{L}$, and T Dwarfs at http://spider.ipac.caltech.edu/staff/davy/ARCHIVE/, are the work of Robert Hurt and were supported through a grant to HST Proposal \#08563 provided by the Space Telescope Science Institute, which is operated by the Association of Universities for Research in Astronomy, Inc., under NASA contract NAS5-26555. The renderings of Figure 2 would not have been possible without the assistance of Mark Marley, who provided guidance on the visible appearance of these objects using the latest results from model atmosphere calculations. The author would also like to thank Adam Burrows for providing the spectral model plotted in Figure 3.

\section{References}

Burgasser, A. J., et al. 2000, ApJL, 531, 57.

Burrows, A., et al. 1997, ApJ, 491, 856.

Chabrier, G. 2002, ApJ, 567, 304.

Kirkpatrick, J. D., et al. 1999, ApJ, 519, 802.

Reid, I. N., et al. 1999, ApJ, 521, 613. 